\section{Suppression of "rubral" tremor with levodopa}

Lesions of the projection pathways from the dentate to the ventrolateral thalamic nucleus in the environs of the red nucleus produce an intractable "rubral" tremor, which has a frequency of around $4 \mathrm{~Hz}$ and is present similarly at rest and through posture and action. Rubral tremor is thought to be related to the resting tremor of Parkinson's disease, for they are indistinguishable in frequency ${ }^{1}$ and both entail synchronous rhythmical activity in VL thalamus neurones. ${ }^{2}$ Levodopa has a variable suppressive effect on Parkinsonian resting tremor, ${ }^{3}$ and the rationale for its action is that it repletes diminished concentrations of dopamine in the striatum thereby restoring its inhibitory influence on the thalamus. ${ }^{4}$

Because of the similarities between rubral and Parkinsonian tremor we evaluated levodopa treatment in a patient with a mesencephalic angioma producing intractable tremor, the lesion being so circumscribed that it provided a paradigm of rubral tremor. Previously levodopa has been shown to suppress tremor arising from electrolytic lesions of the dentate and interpositus nuclei in monkeys (Macaca mulatta). ${ }^{5}$

\section{Case report}

The patient, a 51-year-old, right-handed man, was well until 1957 when he had sudden onset of severe headache followed by loss of consciousness. Subsequent investigations confirmed that he had had a subarachnoid haemorrhage. Angiography showed an arteriovenous malformation arising from near to the tip of the basilar artery in the interpeduncular fossa with a large anomalous draining vein passing through the mid brain to the right of the mid line. His permanent neurological deficits were a fixed, dilated right pupil with impaired ocular movement on the right in all directions save for abduction. He had a mild left-sided spastic hemiparesis. Six weeks after the acute episode he developed involuntary movements of the left hand which became more severe over the next six months and progressed to affect the whole arm and, to a lesser extent, the left leg. The tremor of the arm was a large amplitude, violent tremor at rest, more appreciable distally, which persisted and was exaggerated throughout voluntary movement and posture. Unrestrained the tremor could be self-injuring and necessitated wearing a large restraining harness. The tremor of the leg was intermittent and less severe and did not significantly impair gait. Because of the site of the lesion he was considered unsuitable for surgical treatment and during the years various drugs, including tetrabenzine, diazepam, clonazepam, and orphenadrine, were tried without effect.

The patient was laid on a couch with his left arm outstretched, the fingers strapped down to prevent the arm from moving wildly. An acceleromete was mounted on the dorsum of his hand and the signals were fed to a digital computer which measured the frequency and amplitude of the tremor. During one hour of baseline recordings the tremor had a constant frequency of $3.8 \mathrm{~Hz}$ and an average peak acceleration of $1 \mathrm{~g}\left(\mathrm{~g}=981 \mathrm{~cm} / \mathrm{s}^{-2}\right)$. He was then given a single oral dose of levodopa (Sinemet 275) and observed for an hour. His vigilance was maintained with conversation and arithmetic. After five minutes his tremor began to decrease in amplitude until by the end of the hour the mean peak acceleration was one-eightieth of the initial value (figure), and remained so for five hours. In view of this response he was started on incremental doses of Sinemet, building up to $220 \mathrm{mg}$ three times a day. At this dosage he was able, for the first time in 20 years, to dispense with his harness, and has continued to do so since.

\section{Comment}

This case shows that levodopa can suppress a tremor similar to that of Parkinson's disease but without basal ganglia lesions, and that its suppressive effect is greater than is usual in Parkinson's disease. This

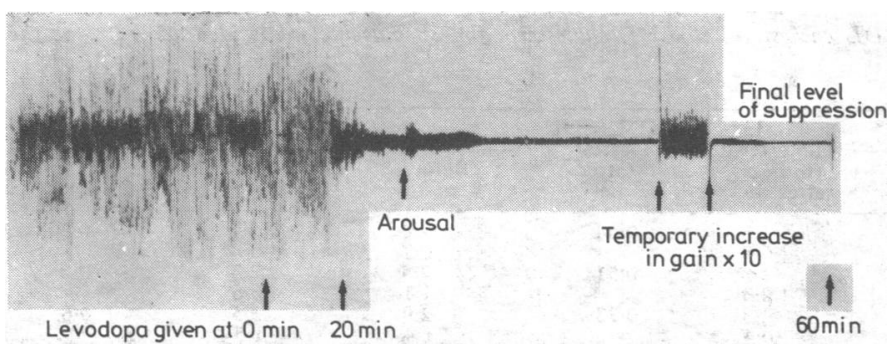

Continuous two-hour accelerometric recording in case of tremor suppressed with levodopa. Time scale is not linear, being compressed and expanded to show important events indicated. implies that the sites of action of levodopa are more widespread than is thought. In neurological practice similar tremors are most commonly seen in multiple sclerosis with brain stem plaques and in brain stem vascular disease. Unfortunately, when there are multiple lesions or widespread damage from a single lesion our experience is that treatment with levodopa is neither as successful nor predictable.

1 Holmes G. On certain tremors in organic cerebral lesions. Brain 1904 ;27: 360-75.

2 Struppler A, Velho-Groneberg P, Claussen M. Clinic and pathophysiology of tremor. In: Birkmayer W, Hornykiewicz $\mathrm{O}$, eds. Advances in parkinsonism. Philadelphia: W B Saunders, 1974:287-302.

3 Markham CM, Treciokas LJ, Diamond SG. Parkinson's disease and levodopa. A five year follow-up and review (medical progress). West $\mathcal{f}$ Med $1974 ; 121$ :188-206.

4 Evarts EV. Neurophysiological mechanisms in Parkinson's disease. In: Birkmayer W, Hornykiewicz $\mathrm{O}$, eds. Advances in parkinsonism. Philadelphia: W B Saunders, 1974:37-54.

5 Goldberger ME, Growden JH. Tremor at rest following cerebellar lesions in monkeys: effect of $\mathrm{L}$-dopa administration. Brain Res 1971 ;27:183-7.

(Accepted 14 August 1980)

National Hospital, Queen Square, London WC1N 3BG, and MRC Hearing and Balance Unit, Institute of Neurology, National Hospital, London WC1N 3BG

L J FINDLEY, MB, MRCP, senior registrar

M A GRESTY, BA, PHD, senior scientist, MRC department of hearing and balance

\section{Adverse reaction to bupivacaine: complication of intravenous regional analgesia}

Bupivacaine has been suggested to be the agent of choice for intravenous regional analgesia because of its effectiveness and freedom from adverse reactions. ${ }^{12}$ I report a case of unexpected neurotoxicity after intravenous regional analgesia with bupivacaine.

\section{Case report}

A healthy 15-year-old West Indian boy weighing $63 \mathrm{~kg}$ presented with a displaced epiphyseal fracture of the distal end of radius. He was taking no medication, had no known allergies, and was sickle-cell negative. No premedicant drugs were given. A tourniquet cuff was applied to the upper part of the arm. A $23 \mathrm{G}$ indwelling needle was placed in a vein on the dorsum of the right hand. The arm was exsanguinated by raising it, the cuff was inflated to a pressure of $200 \mathrm{~mm} \mathrm{Hg}$, and $1.5 \mathrm{mg} / \mathrm{kg} 0.25 \%$ bupivacaine was injected through the indwelling needle (total dose $95 \mathrm{mg}$ ). Complete analgesia resulted and the fracture was reduced and immobilised without any discomfort to the patient.

Forty minutes after the injection of anaesthetic the cuff was released without reinflation. Within 45 seconds of releasing the cuff the patient began to talk excitably with a continuous dialogue of seemingly unrelated personal events. He was completely unresponsive to commands and apparently unaware of his surroundings. His speech was rapid, without slurring, and was concerned with events and grievances at his place of work, his sporting skills, and other personal problems. At the same time he developed irregular twitching of both arms and legs and, to a lesser degree, of the trunk. This hyperexcitable state was considered to be preictal, and after three minutes diazepam $10 \mathrm{mg}$ was given intravenously to reduce the likelihood of a major convulsion. The symptoms persisted. After 12 minutes another $10 \mathrm{mg}$ diazepam was given. The twitching diminished but the confused speech continued. Forty-five minutes after deflation of the cuff the patient was restless and talking irrationally and excitedly. Twitching was now very infrequent. He was sedated with $100 \mathrm{mg}$ chlorpromazine given intravenously in $25-\mathrm{mg}$ increments. He slept soundly until the next morning and had no further twitching. His heart rate, blood pressure, and respiratory rate and pattern remained stable throughout. He was discharged the next day.

\section{Comment}

Presumably bupivacaine was released into the circulation after the cuff was deflated, since the symptoms that followed immediately were similar to the neurotoxic effects of other intravenous local anaesthetics. ${ }^{3}$ Tremor and convulsions have been reported after epidural block with 